\title{
The test-retest reliability of free associations following successive associations'
}

\author{
S. JOYCE BROTSKY, San Fernando Valley \\ State College, Northridge, Calif. 91324, and \\ MARIGOLD L. LINTON, San Diego State \\ College, San Diego, Calif. 92115
}

A word-association test (WAT) consisting of four randomizations of 68 different words was given to $72 \mathrm{Ss}$. The retest, a fifth randomization of the word set, was given 4 weeks later. The tendency to give identical associates on the WAT and retest was related to the dominance of the normative primary. Ss who gave many primaries tended to give the same associate repeatedly. When a reproductive response strategy is not limited by instructions, some Ss may repeat the same response to a given stimulus each time it is presented if that stimulus has a highly dominant primary response.

Studies have been conducted (Brotsky, Butler, \& Linton, 1967; Brotsky \& Linton, $1967 \mathrm{~b}, \mathrm{c})$ to determine the conditions under which Ss give the same verbal response to a given verbal stimulus on two separate occasions. The results have indicated that the probability of occurrence of an identical associate (IA) can be predicted from the shape of the normative response hierarchy of the stimulus. That is, when a stimulus elicited the same response from many $S s$ in a free-association test (steep normativeresponse hierarchy), that response tended to be given by a particular $S$ each time the stimulus word was presented, resulting in few different responses (DR) and many IAs during the retest. When a stimulus elicited many DRs from normative Ss (flat normative-response hierarchy), there was little consistency in the responses given by a particular $S$ when the stimulus was presented on later occasions, resulting in many DRs and few IAs on the retest. Moreover, Ss who gave many popular responses $(\mathrm{P})$ tended to be consistent in their responses, producing many IAs on the two response occasions provided.

The present study further investigated test-retest reliability (TRR) using the word association format known as the method of successive associations (Cramer, 1968) in order to elicit from each $S$ a hierarchy of four discrete responses to each stimulus word.

\section{SUBJECTS}

The Ss were 72 undergraduates between the ages of 17 and 29. All Ss had been born in continental United States and had learned
English as their first language. The Ss participated as part of the requirements for a summer-session course in introductory psychology at San Diego State College or San Fernando Valley State College. The data were collected by the authors from three separate intact classes during class time. STIMULUS MATERIAL

DP and DR were determined for each of the 68 words from norms developed by the authors (Brotsky \& Linton, 1967a). Few Ss (8\%) gave the normative primary (P) to either the word MARK or the word SCENE, while the $P$ with the greatest degree of commonality was THREAD, the associate given by $93 \%$ of the Ss to SPOOL. The mean DP of the word set was $69 \%$. INK was so dominant a $\mathrm{P}$ to BLOTTER that there were only 19 DRs, while MADE, a low DP stimulus, elicited the largest number of DRs (95).

The language frequency (LF) of the stimuli were assessed (Thorndike \& Lorge, 1944). Twenty-three words had LF counts of 38 per million or less; 23 words had LF counts of from 40 to 50 occurrences per million; 22 stimuli were $A$ or AA words.

The WAT booklet consisted of four different randomizations of the 68 stimulus words, 25 per page, each followed by a line on which $S$ was instructed to write his response. The retest was a fifth randomization of the word set. All Ss received the stimuli in the same serial order.

\section{PROCEDURE}

Standard free association instructions were given. The Ss were informed prior to the WAT that some of the words might be repeated one or more times; however, the first word that came to mind was to be given without attempting to recall previous responses. The retest was administered 4 weeks after the WAT, and no statement was made regarding the relationship between the two sessions. The $\mathrm{E}$ wrote the time on the blackboard at 1-min intervals, and $S$ was instructed to write the time of completion at the bottom of his booklet. The data were analyzed within-words and within-Ss.

RESULTS AND DISCUSSION

Within-Words Analysis (Across Ss)

\section{WAT Data}

None of the $72 \mathrm{Ss}$ gave the $\mathrm{P}$ to the word MARK on the first WAT trial, while SPOOL elicited the greatest number of Ps (62). On the first WAT trial $36.3 \%$ of the responses were Ps, but little variation was shown in the percentage of Ps given on each of the four
WAT trials (35\%-36\%). This finding was not in accord with the prediction that there would be a decreasing number of Ps on successive trials. The substantial correlation between DP and the number of Ps given on the first WAT trial $(r=.90, \mathrm{df}=66, \mathrm{p}<.01)$ indicated that the language habits of the present sample and the normative group were similar.

Retest Data

The word BLOCK and the word MUSIC elicited Ps from only three Ss, while $76 \%$ of the Ss gave the highly dominant THREAD to the stimulus word SPOOL. When the response that was given by a particular $S$ on the retest was identical to the response that he had previously given on one of the WAT trials, an IA was said to have occurred. All variations in spelling other than the plural were taken as separate entries. The number of IAs given in the first WAT trial and on the retest ranged from $8 \%$ for MUSIC to $83 \%$ for SPOOL. When an IA was defined in terms of an identical response on the first WAT trial and on the retest, the mean TRR was $44 \%$. The number of IAs given on any of the four WAT trials and on the retest ranged from $16 \%$ for MUSIC to $82 \%$ for BLOTTER. When an IA was defined in this manner, the mean TRR was $46 \%$.

The high positive correlation $(r=.87$, $\mathrm{df}=66, \mathrm{p}<.01$ ) between the number of Ps on the first WAT trial and the number of IAs on the first WAT trial and on the retest constituted a replication of the results of two earlier studies (Brotsky \& Linton, $1967 \mathrm{~b}, \mathrm{c})$. The correlation between DP and the number if IAs on the first WAT trial and the retest $(r=.86, \mathrm{df}=66, \mathrm{p}<.01)$ suggested that predictions of TRR can be made on the basis of normative data. The positive correlation $(r=.87)$ between DP and the number of IAs occurring on any of the four WAT trials and the total number of Ps on the retest $(r=.95)$ provided further evidence of the stability of the relationship between TRR and the commonality of the P.

In accordance with earlier results, a negative correlation was found between the LF of the stimulus word and the number of IAs on the first WAT trial and on the retest $(\mathrm{r}=-.40, \mathrm{df}=66, \mathrm{p}<.01)$. Moreover, there was a negative correlation between LF and the number of IAs which were given on any of the four WAT trials and the retest $(\mathrm{r}=-.38, \mathrm{df}=66, \mathrm{p}<.01)$. Although the correlation between LF and total number of Ps given on all four WAT trials $(r=-.20)$ was not statistically reliable, the correlation between LF and the number of Ps on the retest $(r=-.26, \quad$ df $=66, .01>p<.05)$ approached statistical significance.

The relationship between the WAT trial on which a response occurred and the kind of response given was explored. Although the retest response had occurred on at least 
one of the four WAT trials $61 \%$ of the time, the probability of occurrence of an IA did not vary monotonically as a function of trial number ( .33 for the first trial to .29 for the second trial, with intermediate values on the third and fourth trials).

Within-Ss Analysis (Across-Words) WAT Data

In order to determine the response pattern of the individual Ss, a number of indices were used. The mean number of $P_{s}$ given to the 272 stimuli on the WAT was 95.8 (range $=31-150$ ). The mean number of stimulus words on the WAT for which a $P$ was given in at least one of the four WAT trials was 33.6 (range $=16-44$ ). The mean number of DRs on the WAT was 136.8 (range $=77-228$ )

Retest Data

The mean number of Ps on the retest was 27 (range $=8-40)$. The mean number of IAs that occurred on the first WAT trial and on the retest was 30.5 (range $=14-47$ ). The mean number of stimuli to which the same response occurred on any of the four WAT trials as well as the retest was 41.4 (range $=27-54$ ).

In the absence of instructions to the contrary, some Ss adopted the strategy of repeating the same response to two or more of the four presentations of a given stimulus in the WAT. The mean number of IAs in at least two trials of the WAT as well as the retest was 33.8 (range $=14-51$ ). The mean number of IAs in at least three trials of the WAT and on the retest was 26.7 (range $=4-46$ ). The mean number of IAs given on all four trials of the WAT and on the retest was 18.2 (range $=0-44$ ). If $S$ adopted the strategy of giving the same response to each word on the four trials of the WAT as well as the retest he would have had 272 IAs. In the present sample, the mean number of IAs was 118.4 (range = 49-196).

Those Ss who tended to emit Ps tended to repeat their response or adopt what Applebaum (1960) has called a "reproductive" strategy. The correlation between number of Ps on the WAT and the tendency to give IAs on two or more WAT trials was highly reliable $(\mathrm{r}=.77, \mathrm{df}=70, \mathrm{p}<.01)$. The product moment correlation between the total number of Ps on the WAT and the production of IAs on all four trials of the WAT was $.75(\mathrm{df}=70, \mathrm{p}<.01)$. The correlation between number of Ps on the retest and the tendency to give an IA on all four trials of the WAT was $.60(\mathrm{df}=70$, $\mathrm{p}<.01$ ). Those $\mathrm{Ss}$ who tended to give a large proportion of Ps and IAs gave few DRs. For example, there was a strong negative correlation $(r=-.72)$ between DR and total number of Ps on the WAT. Moreover, there was a strong negative correlation $(r=-.67)$ between DR and number of IAs on the first WAT trial and the retest.

Although there was little difference in either the number of Ps or the number of IAs given on the four trials of the WAT, there were significantly more Ps given to the stimuli on the retest than on any of the four WAT trials. Using a $t$ test for correlated measures, there was a significant difference between the number of Ps on the first WAT trial (36.3\%) and the number of Ps given in the retest $(39.5 \%)[t(67)=2.90, p<.01]$. This finding was in accord with the data reported by Weintraub, Silverstein, \& Klee (1960) whose normal Ss, displaying what was called "corrective" behavior, gave significantly fewer populars in a WAT than in a re test administered 1 week later.

\section{GENERAL COMMENTS}

The present study, while replicating the results of earlier research which demonstrated a strong relationship between DP and TRR (Brotsky \& Linton, 1967b, c), failed to produce a hierarchy of four discrete responses from each of the Ss to each of the 68 words. This was due to the pronounced tendency on the part of Ss to emit the same response to two or more presentations of a given stimulus. This strategy was related both to the shape of the normative response hierarchy of the stimuli and Ss' tendency to respond with Ps. Thus, in the absence of contrary instructions, those Ss who tended to give a large number of Ps (popular responders) tended to emit the same $P$ at the intervals ranging from minutes to days or weeks. When a stimulus had no highly dominant $P$, there were many competing DRs, and reproduction occurred less frequently. Even those $S s$ who rarely gave Ps (idiosyncratic responders) tended to reproduce Ps when the stimulus had a very strong popular. With stimuli having weaker populars, however, the idiosyncratic responders tended not to repeat their responses, producing a low degree of TRR.

\section{REFERENCES}

APPLEBAUM, S. A. Automatic and selective processes in the word associations of brain damaged and normal subjects. Journal of Personality, 1960, 28, 64-72.

BROTSKY, S. J., BUTLER, D. C., \& LINTON, M. L. Association time, commonality, and the test-retest reliability of free association responses. Psychonomic Science, 1967, 9 319-320.

BROTSKY, S. J., \& LINTON, M. L. The San Fernando Valley State College word association norms. Technical Report No. 1, 1967a, San Fernando Valley State College, Research Grant 4.259.01, San Fernando Valley State College Foundation.

BROTSKY, S. J., \& LINTON, M. L. The test-retest reliability of free association norms. Psychonomic Science, $1967 \mathrm{~b}, 8,425426$.

BROTSKY, S. J., \& LINTON, M. L. The test-retest reliability of free associations following continued associations. Psychonomic Science, $1967 \mathrm{c}, 9,85-86$

CRAMER, P. Word association. New York: Academic Press, 1968.

THORNDIKE, E. L., \& LORGE, I. The teacher's word book of 30,000 words. New York: Teacher's College, Columbia University, 1944. WEINTRAUB, W., SILVERSTEIN, A., \& KLEE, G. D. The "correction" of deviant responses on a word association test. Archives of General Psychiatry, 1960, 3, 17-20.

\section{NOTE}

1. This research was supported in part by a research grant to the first author from the San Femando Valley State College Foundation and by a faculty research award to the second author from the San Diego State College Foundation. The report was written during the first author's tenure as NIH Special Fellow in Human Development, Harvard University.

\section{The effect of pronunciation practice on anagram difficulty}

ROGER L. DOMINOWSKI, University of Illinois at Chicago Circle, Chicago, Ill. 60680

A comparison was made of the solution difficulty of anagrams differing in pronounceability but having the same solutions, either with or without prior pronunciation practice with the anagrams. Anagrams rated easier to pronounce had faster promunciation speeds and lower solution probabilities. Pronunciation practice increased reading speed and reduced solution probability. It was suggested that pronounceability reflects $S s^{\prime}$ tendencies to deal with an anagram as a unit rather than as a sequence of letters, and the results were related to comparisons of word and nonsense anagrams.

Hebert \& Rogers (1966) reported that anagrams which are easy to pronounce (EP) are more difficult to solve than anagrams that are hard to pronounce (HP). However, the design of their study did not permit an unambiguous conclusion concerning the effect of anagram pronounceability since EP and HP anagrams had different solution words. Other research has indicated that individual solution words exhibit consistent differences in difficulty even when their respective anagrams are radically changed (e.g., Dominowski, 1967). Thus, it is possible that the difference between pronounceability conditions was due, to some unknown degree, to differences in solution words. A major purpose of the present study was to compare anagrams varying in pronounceability but having the same solution words. 